\title{
Semitransparent organic solar cells with hybrid monolayer graphene/metal grid as top electrodes
}

\author{
Peng Lin, ${ }^{1}$ Wallace C. H. Choy, ${ }^{1, a)}$ Di Zhang, ${ }^{1}$ Fengxian Xie, ${ }^{1}$ Jianzhuo Xin, ${ }^{2}$ \\ and C. W. Leung ${ }^{2}$ \\ ${ }^{1}$ Department of Electrical and Electronic Engineering, The University of Hong Kong, Pokfulam Road, \\ Hong Kong, China \\ ${ }^{2}$ Department of Applied Physics, The Hong Kong Polytechnic University, Hung Hom, Kowloon, \\ Hong Kong, China
}

(Received 1 January 2013; accepted 12 March 2013; published online 20 March 2013)

\begin{abstract}
Hybrid transparent monolayer graphene/metal grid is proposed as top electrode of semitransparent organic solar cells. The hybrid electrode using gold grid on flexible polyethylene terephthalate substrate shows very low sheet resistance of $22 \pm 3 \Omega / \square$ and high optical transmittance of $81.4 \%$, which is comparable to conventional indium tin oxide/glass electrode. Using lamination process, the layer of poly(3,4-ethylenedioythiophene):poly(styrenesulfonate) doped with D-sorbitol plays an important role in the electrical performance of the laminated devices. In addition, the devices show best power convention efficiency of $3.1 \%$ and fill factor of $55.0 \%$, which are much better than those of similar graphene-based semitransparent organic solar cells. (C) 2013 American Institute of Physics. [http://dx.doi.org/10.1063/1.4798254]
\end{abstract}

Graphene, a two-dimensional (2D) sheet of hexagonal lattice carbon material, has attracted great attention in different research areas. ${ }^{1}$ Due to its outstanding optical, electrical, and mechanical properties, graphene has been widely adopted in organic photovoltaics, in which graphene is used as active layer, ${ }^{2}$ charge transport layer, ${ }^{3}$ and transparent electrode. ${ }^{4}$ Especially for transparent electrode, graphene has shown great advantages over indium tin oxide (ITO) because ITO has the issues of limited indium source and poor transparency in near infrared region. ${ }^{5}$ In addition, the brittle feature of ITO has limited its application in flexible photovoltaic devices. ${ }^{6}$ On the contrary, graphene shows excellent flexibility and mechanical strength, which make it more favorable in the development of "plastic electronics."7

However, one of the critical issues for the application of graphene as transparent electrode is the poor conductivity compared with ITO or metal electrodes. As a result, the power convention efficiency (PCE) of such graphene-based device is relatively low. ${ }^{8-10}$ For example, Acro et al. demonstrated organic solar cells (OSCs) with PCE of only $1.18 \%$ based on chemical vapor deposition (CVD) graphene electrode. ${ }^{8}$ Meanwhile, stacked multi-layer graphene ${ }^{11,12}$ and chemically doped graphene films ${ }^{13,14}$ have been used to obtain more conductive graphene electrodes. Wang et al. reported OSCs with layer-by-layer stacked graphene anode, in which PCE improved to $2.5 \% .^{11}$ However, layer-by-layer method is relative time-consuming, and the graphene films may be easily destroyed in the process, which may not be suitable for large-area film fabrication. Lee et al. demonstrated OSCs with $\mathrm{HNO}_{3}$-doped graphene electrode, which showed PCE of about 2.5\%. ${ }^{13}$ However, the stability is a challenge for such chemically doped graphene films.

Very recently, graphene has been considered as the potential candidate for the top electrode of semitransparent

${ }^{\text {a)} E l e c t r o n i c ~ m a i l: ~ c h c h o y @ e e e . h k u . h k . ~}$
OSCs,${ }^{15,16}$ which have interesting applications like power windows for buildings and automobiles. ${ }^{17}$ In this work, hybrid transparent monolayer graphene/metal grid is proposed as the top electrode of semitransparent OSCs. Our results show that the hybrid electrode is comparable to ITO and shows very good stability. In addition, lamination process is used for the fabrication of devices, and the advantages will be discussed. The OSCs fabricated by lamination process are semitransparent, self-packaged, and highly efficient, which exhibit good potential in large-area roll-to-roll manufacturing applications.

The semitransparent OSCs based on poly(3-hexylthiophene):[6,6]-phenyl C61-butyric acid methyl ester (P3HT: PCBM) were fabricated by lamination process. The device structure was glass/ITO/TiO $2 / \mathrm{P} 3 \mathrm{HT}$ :PCBM/PEDOT:PSS/monolayer graphene/Au grid/PET. Two parts of the devices (P3HT:PCBM/TiO $/ /$ ITO/glass and PEDOT:PSS/monolayer graphene/Au grid/PET) were fabricated separately and then laminated together. For the fabrication of P3HT:PCBM/ $\mathrm{TiO}_{2} / \mathrm{ITO} / \mathrm{glass}, \mathrm{TiO}_{2}(20 \mathrm{~nm})$ and P3HT:PCBM $(220 \mathrm{~nm})$ were optimized by solution process, which was described in our previous work. ${ }^{18}$ For the fabrication of PEDOT:PSS/ monolayer graphene/Au grid/PET, first, Au grid was fabricated on flexible PET substrate by photolithography with lift-off technique. ${ }^{19}$ A thin layer of chromium $(\mathrm{Cr}, 5 \mathrm{~nm})$ was used as the adhesion layer. The thickness of Au grid was $50 \mathrm{~nm}$. Different grid dimensions $(50 \mu \mathrm{m} \times 50 \mu \mathrm{m}, 100 \mu \mathrm{m}$ $\times 100 \mu \mathrm{m}, 200 \mu \mathrm{m} \times 200 \mu \mathrm{m}$, and $300 \mu \mathrm{m} \times 300 \mu \mathrm{m})$ with same grid line width $(10 \mu \mathrm{m})$ were designed. After forming the metal grids, monolayer graphene grown on copper foils by CVD method (from Graphene Supermarket) was transferred to the top of Au grid/PET substrate by traditional solution method. ${ }^{15}$ Then PEDOT:PSS (Baytron AI 4083) doped with $50 \mathrm{mg} / \mathrm{ml} \mathrm{D}$-sorbitol was spin-coated on graphene/Au grid/PET substrate. After the two parts of the devices were fabricated, a lamination technique was conducted to obtain final devices. Both substrates were heated at $120^{\circ} \mathrm{C}$, and a 

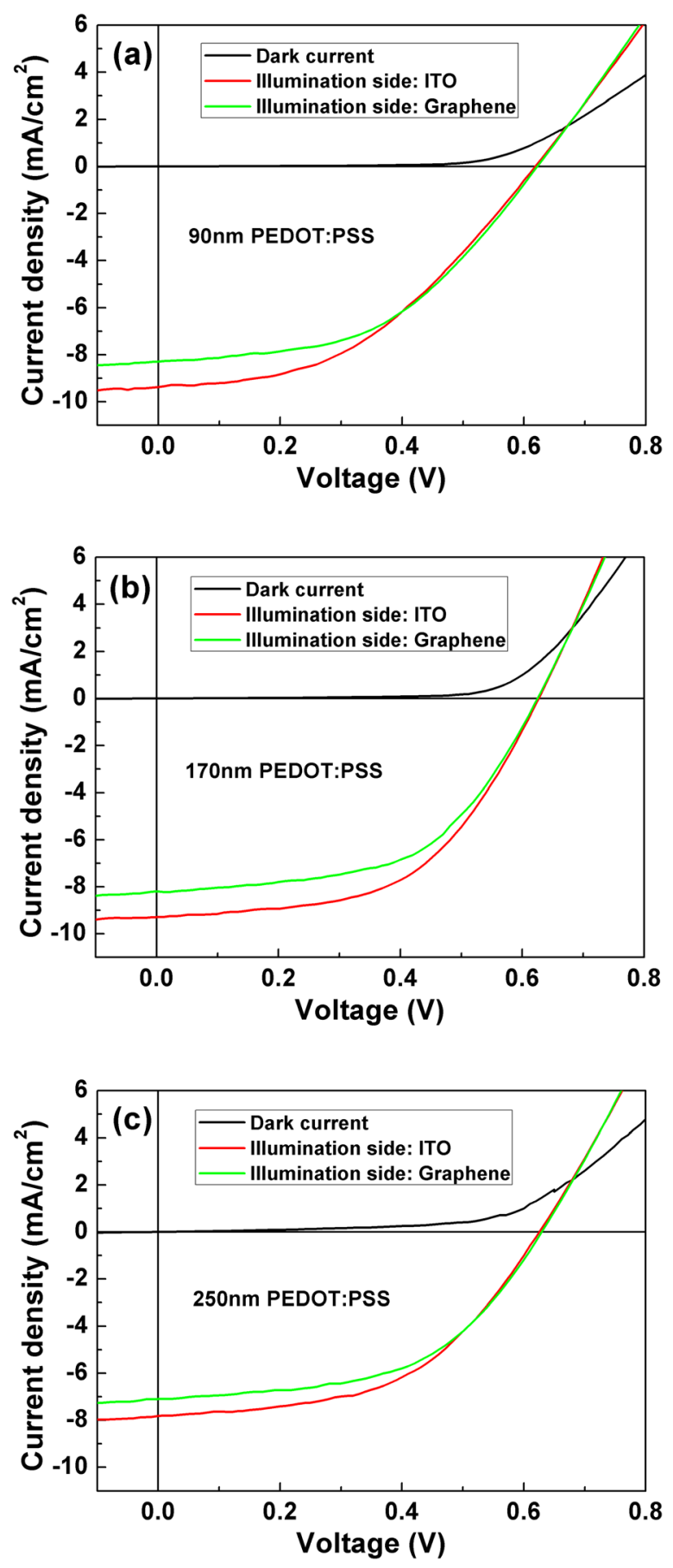

FIG. 1. $J-V$ characteristics of OSCs measured under illumination from both sides of the semitransparent devices with different thickness of PEDOT:PSS: (a) $90 \mathrm{~nm}$, (b) $170 \mathrm{~nm}$, (c) $250 \mathrm{~nm}$. The grid size of Au grid is $200 \mu \mathrm{m} \times 200 \mu \mathrm{m}$.

plastic rod was rolled with proper pressure on the PET substrate to remove air bubbles, following $5 \mathrm{~min}$ final heat treatment on the hotplate.
Current density $(J)$-voltage $(V)$ characteristics of the devices are measured by a Keithley 2635 sourcemeter under illumination of $100 \mathrm{~mW} / \mathrm{cm}^{2}$ from ABET AM 1.5G solar simulator. The area of each device is around $0.08-0.1 \mathrm{~cm}^{2}$. Figure 1 shows the $J-V$ characteristics of OSCs with different thickness of PEDOT:PSS measured under illumination from both sides of the semitransparent devices. The electrical performances are summarized in Table I. The series resistance $\left(R_{\mathrm{S}}\right)$ is calculated from the inverse slope of the illuminated $J-V$ characteristics at $1.0 \mathrm{~V}$. The grid size of Au grid is $200 \mu \mathrm{m} \times 200 \mu \mathrm{m}$. We can find that the best electrical performance is obtained when the thickness of PEDOT:PSS is $170 \mathrm{~nm}$, which shows PCE of $3.1 \%$ from ITO side and $2.8 \%$ from graphene side. The different electrical performance from two sides of the device is mainly due to the different optical transmittance of the two electrodes as shown in Fig. 2(a). Incident-photon-to-electron conversion efficiency (IPCE) of the device also indicates the different performance from two sides, as shown in Fig. 3. The control experiment shows that the device with monolayer graphene as top electrode (without Au grid) only exhibits PCE of about $0.8 \%$. We can deduce that the high PCE of the OSCs with monolayer graphene/Au grid hybrid electrodes is attributed to three key factors.

First, the good quality of the hybrid monolayer graphene/Au grid electrodes makes the most significant contribution to the high electrical performance of the OSCs. The sheet resistance and optical transmittance of the monolayer graphene/Au grid hybrid electrodes on PET substrates are characterized by a four-point probe and spectroscopic ellipsometry, respectively. With different grid sizes, the sheet resistance shows a value of $7-30 \Omega / \square$, while the optical transmittance is about $66 \%-84 \%$ (at $550 \mathrm{~nm}$ ). It is found that the conductivity of the hybrid electrode decreases with the increase of $\mathrm{Au}$ grid size, while the optical transmittance increases when the Au grid size increases. The optimized performance is obtained when the grid size is $200 \mu \mathrm{m}$ $\times 200 \mu \mathrm{m}$, as shown in Fig. 2 . The hybrid electrode exhibits a sheet resistance as low as $22 \pm 3 \Omega / \square$ and an optical transmittance of $81.4 \%$ (at $550 \mathrm{~nm}$ ), which is comparable to ITO/ glass electrode $(16.5 \pm 0.2 \Omega / \square$ with transmittance of $82.8 \%$ at the same wavelength).

Compared with monolayer graphene electrode with a sheet resistance of $1.2 \pm 0.3 \mathrm{k} \Omega / \square$ in our work, the hybrid electrode shows highly improved conductivity. Furthermore, the hybrid electrode is more stable and repeatable than those chemically doped graphene films such as acid doping. Most importantly, the hybrid electrode can be fabricated on various substrates such as glass and flexible PET, which makes it more favorable for practical applications. Consequently, the

TABLE I. Summary of electrical performances of OSCs with different thickness of PEDOT:PSS.

\begin{tabular}{lccccccc}
\hline \hline Thickness of PEDOT:PSS & Illumination side & $J_{\mathrm{sc}}\left(\mathrm{mA} / \mathrm{cm}^{2}\right)$ & $V_{\mathrm{oc}}(\mathrm{V})$ & FF $(\%)$ & PCE $(\%)$ & $R_{\mathrm{S}}\left(\Omega \mathrm{cm}^{2}\right)$ & $R_{\mathrm{SH}}\left(\Omega \mathrm{cm}^{2}\right)$ \\
\hline \multirow{2}{*}{$90 \mathrm{~nm}$} & ITO & 9.37 & 0.62 & 43.3 & $2.52 \pm 0.14$ & 27.6 & 685 \\
& Graphene & 8.29 & 0.62 & 48.1 & $2.47 \pm 0.14$ & 25.9 & 649 \\
\multirow{2}{*}{$170 \mathrm{~nm}$} & ITO & 9.30 & 0.63 & 53.2 & $3.11 \pm 0.21$ & 13.5 & 1147 \\
& Graphene & 8.20 & 0.62 & 55.0 & $2.79 \pm 0.19$ & 14.8 & 716 \\
\multirow{2}{*}{$250 \mathrm{~nm}$} & ITO & 7.83 & 0.63 & 50.3 & $2.48 \pm 0.17$ & 20.1 & 470 \\
& Graphene & 7.10 & 0.63 & 52.5 & $2.35 \pm 0.16$ & 19.7 & 569 \\
\hline \hline
\end{tabular}



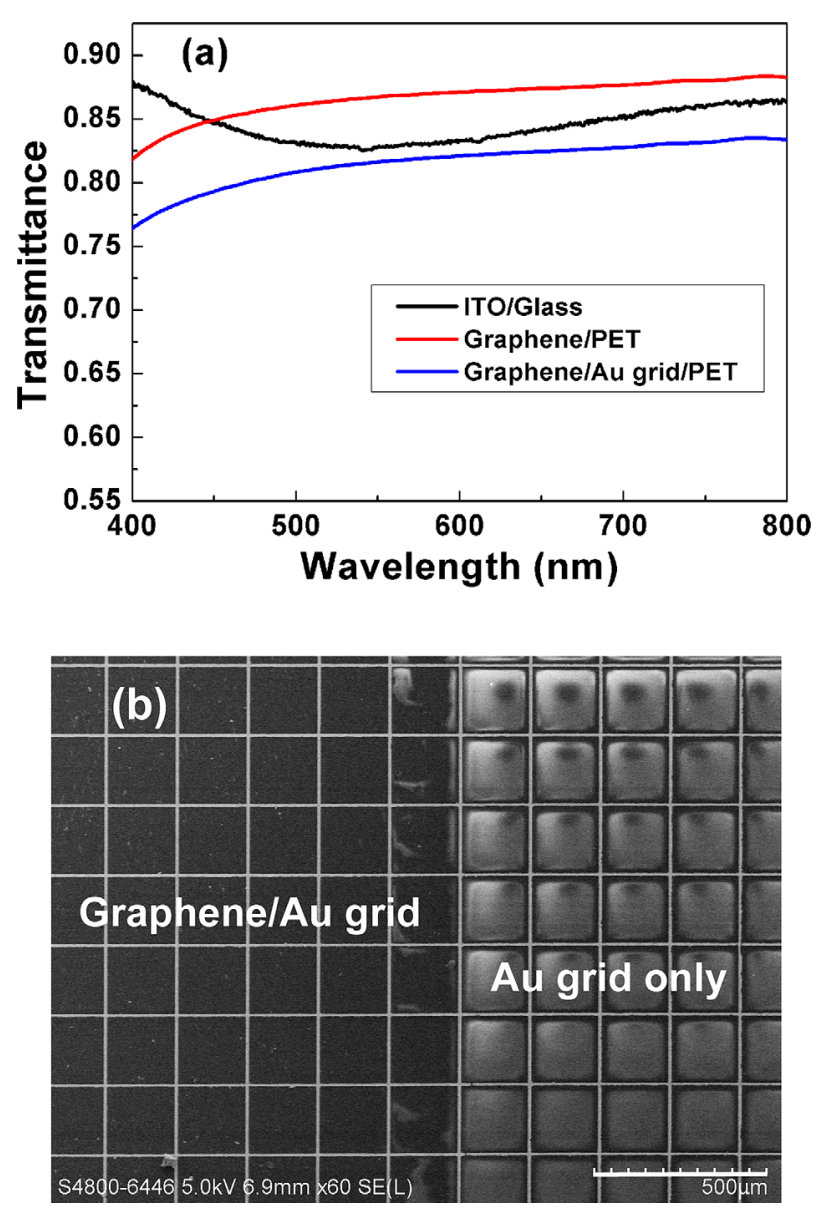

FIG. 2. (a) Optical transmittance of three different electrodes: ITO/glass, monolayer graphene/PET, and monolayer graphene/Au grid/PET. (b) SEM image of the monolayer graphene/Au grid hybrid transparent electrode. The grid size of Au grid is $200 \mu \mathrm{m} \times 200 \mu \mathrm{m}$.

monolayer graphene/metal grid hybrid transparent electrodes show excellent potential for the application in OSCs.

Second, the lamination process in our work has many advantages in the fabrication process of OSCs with graphene-based top electrode, which is of benefit to the electrical properties and thus PCE of the OSCs. Compared with the solution process of transferring graphene as top electrode, the lamination process is harmless to the polymer

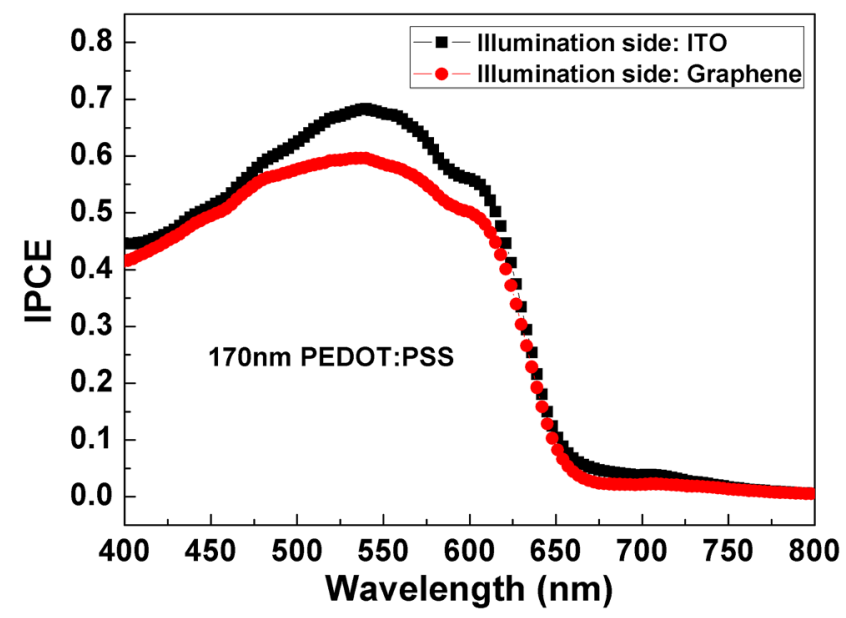

FIG. 3. IPCE of OSC measured under illumination from both sides of the semitransparent device with $170 \mathrm{~nm}$ thick PEDOT:PSS. active layer, which makes the device more stable and competitive. Large-area device fabrication through a roll-to-roll process becomes possible if both substrates are plastic and the devices are also self-packaged. Moreover, thermal vacuum evaporation is not needed after the fabrication of polymer active layer as it is harmful to some kinds of polymer.

Third, the layer of PEDOT:PSS with high quality also contributes to the high electrical performance of the OSCs as it is very important in the lamination process. The PEDOT:PSS solution in our work is modified by doping Dsorbitol because it acts as a very excellent electronic glue and improves the contact of the two substrates. ${ }^{20}$ The optimized concentration of D-sorbitol is $50 \mathrm{mg} / \mathrm{ml}$. It has been reported that spin-coating of PEDOT:PSS on graphene directly is very difficult due to the hydrophobic property of graphene. Several different methods have been used to solve this problem, such as surface modification and employing a hydrophilic interface layer. ${ }^{11,21}$ In our work, a more simple and convenient approach is introduced. The graphene/Au grid/PET substrate is heated at $120^{\circ} \mathrm{C}$ for $2 \mathrm{~min}$ on a hotplate. Then PEDOT:PSS solution is dropped on the hot surface of graphene and kept for $10 \mathrm{~s}$. After that the sample is quickly moved to spin coater and spin-coated at $1500 \mathrm{rpm}$. Finally, the sample is annealed at $120^{\circ} \mathrm{C}$ for $20 \mathrm{~min}$, and a uniform film of PEDOT:PSS with $\sim 90 \mathrm{~nm}$ is obtained. The good performance of OSC dark current indicates the good quality of PEDOT:PSS layer and the excellent contact of the interface between P3HT:PCBM and PEDOT:PSS, as shown in Fig. 1.

Moreover, the thickness of PEDOT:PSS plays an important role in the electrical performance of the laminated devices. The OSCs with one-layer, two-layers, and three-layers of PEDOT:PSS are investigated. The total thickness of PEDOT:PSS is $90 \mathrm{~nm}, 170 \mathrm{~nm}$, and $250 \mathrm{~nm}$, respectively. As shown in Table I, we can find that the short-circuit current density $\left(J_{\mathrm{sc}}\right)$ decreases with the increase of thickness of PEDOT:PSS because the low vertical conductivity in thick PEDOT:PSS will weaken hole transport in PEDOT:PSS layer. With the increasing thickness of PEDOT:PSS, fill factor (FF) increases first, which indicates that higher thickness of PEDOT:PSS improves the contact of the interface between P3HT:PCBM and PEDOT:PSS in lamination process. When the thickness increases to $250 \mathrm{~nm}$, the surface of PEDOT:PSS becomes rough, which leads to bad contact of the interface between P3HT:PCBM and PEDOT:PSS and the consequent decrease of FF. As a result, the optimized electrical performance is obtained when the thickness of PEDOT:PSS is $170 \mathrm{~nm}$, which shows the best PCE of $3.1 \%$ from ITO side and $2.8 \%$ from graphene side. Moreover, the best FF of $55.0 \%$ can be obtained. The lowest series resistance $\left(R_{\mathrm{S}}\right)$ and highest shunt resistance $\left(R_{\mathrm{SH}}\right)$ for the OSCs with $170 \mathrm{~nm}$ thick PEDOT:PSS also indicate the optimized electrical performance of the device. The highly efficient OSCs with the hybrid monolayer graphene/metal grid electrodes are very competitive in the graphene-based semitransparent OSCs.

In this work, we demonstrate that monolayer graphene/ metal grid hybrid electrode can be used as top electrode by using $\mathrm{Au}$ grid. To reduce the cost and make the electrode more favorable for practical application, other metal 
materials like $\mathrm{Cu}$ can be considered. One of the critical issues of such topic is the tuning of work function of the graphene/metal grid hybrid electrode. Furthermore, the hybrid electrode can be also used as cathode, and then both electrodes based on graphene can be developed in semitransparent polymer solar cell. This will benefit the large-area device fabrication through a roll-to-roll process. Further work is in progress on these two topics.

In summary, we have demonstrated hybrid monolayer graphene/Au grid/PET electrode with very low sheet resistance of $22 \pm 3 \Omega / \square$ and high optical transmittance of $81.4 \%$. The hybrid electrode is comparable to ITO/glass electrode and shows very good stability. The semitransparent OSCs have been fabricated by an efficient lamination process. Moreover, a simple and convenient approach is introduced to fabricate PEDOT:PSS on the hydrophobic surface of graphene. We find that PEDOT:PSS layer doped with Dsorbitol plays an important role in the electrical performance of the laminated devices. The device with $170 \mathrm{~nm}$ thick PEDOT:PSS exhibits maximum efficiency of $3.1 \%$ and fill factor of $55.0 \%$, which are much better than those with similar graphene-based semitransparent OSCs.

This work is supported by University Grant Council of the University of Hong Kong (Grant Nos. 10401466 and 201111159062), the General Research Fund (Grant Nos. HKU712010E and HKU711612E), and the RGC-NSFC grant (No. N_HKU709/12) from the Research Grants Council of Hong Kong Special Administrative Region, China. C. W. Leung would like to acknowledge the financial support of PolyU (A-PK38, A-PL51).
${ }^{1}$ K. Geim and K. S. Novoselov, Nature Mater. 6, 183 (2007).

${ }^{2}$ Z. F. Liu, Q. Liu, Y. Huang, Y. F. Ma, S. G. Yin, X. Y. Zhang, W. Sun, and Y. S. Chen, Adv. Mater. 20, 3924 (2008).

${ }^{3}$ J.-M. Yun, J.-S. Yeo, J. Kim, H.-G. Jeong, D.-Y. Kim, Y.-J. Noh, S.-S. Kim, B.-C. Ku, and S.-I. Na, Adv. Mater. 23, 4923 (2011).

${ }^{4}$ X. Huang, Z. Y. Zeng, Z. X. Fan, J. Q. Liu, and H. Zhang, Adv. Mater. 24, 5979 (2012).

${ }^{5}$ A. R. Schlatmann, D. W. Floet, A. Hilberer, F. Garten, P. J. M. Smulders, T. M. Klapwijk, and G. Hadziioannou, Appl. Phys. Lett. 69, 1764 (1996).

${ }^{6}$ Z. Chen, B. Cotterell, W. Wang, E. Guenther, and S.-J. Chua, Thin Solid Films 394, 201 (2001).

${ }^{7}$ S.-I. Na, S.-S. Kim, J. Jo, and D.-Y. Kim, Adv. Mater. 20, 4061 (2008).

${ }^{8}$ L. G. De Arco, Y. Zhang, C. W. Schlenker, K. Ryu, M. E. Thompson, and C. W. Zhou, ACS Nano 4, 2865 (2010).

${ }^{9}$ Z. Y. Yin, S. X. Wu, X. Z. Zhou, X. Huang, Q. C. Zhang, F. Boey, and H. Zhang, Small 6, 307 (2010).

${ }^{10}$ D. Zhang, W. C. H. Choy, C. C. D. Wang, X. Li, L. L. Fan, K. L. Wang, and H. W. Zhu, Appl. Phys. Lett. 99, 223302 (2011).

${ }^{11}$ Y. Wang, S. W. Tong, X. F. Xu, B. Özyilmaz, and K. P. Loh, Adv. Mater. 23, 1514 (2011)

${ }^{12}$ C.-L. Hsu, C.-T. Lin, J.-H. Huang, C.-W. Chu, K.-H. Wei, and L.-J. Li, ACS Nano 6, 5031 (2012).

${ }^{13}$ S. Lee, J.-S. Yeo, Y. S. Ji, C. H. Cho, D.-Y. Kim, S.-I. Na, B. H. Lee, and T. Lee, Nanotechnology 23, 344013 (2012).

${ }^{14}$ Y. U. Jung, S.-I. Na, H.-K. Kim, and S. J. Kang, J. Vac. Sci. Technol. A 30, 050604 (2012).

${ }^{15}$ Z. K. Liu, J. H. Li, Z.-H. Sun, G. A. Tai, S.-P. Lau, and F. Yan, ACS Nano 6, 810 (2012).

${ }^{16}$ Y.-Y. Lee, K.-H. Tu, C.-C. Yu, S.-S. Li, J.-Y. Hwang, C.-C. Lin, K.-H. Chen, L.-C. Chen, H.-L. Chen, and C.-W. Chen, ACS Nano 5, 6564 (2011).

${ }^{17}$ R. Koeppe, D. Hoeglinger, P. A. Troshin, R. N. Lyubovskaya, V. F. Razumov, and N. S. Sariciftci, ChemSusChem 2, 309 (2009).

${ }^{18}$ D. Zhang, W. C. H. Choy, F. X. Xie, and X. C. Li, Org. Electron. 13, 2042 (2012).

${ }^{19}$ Y. Zhu, Z. Z. Sun, Z. Yan, Z. Jin, and J. M. Tour, ACS Nano 5, 6472 (2011).

${ }^{20}$ J. S. Huang, G. Li, and Y. Yang, Adv. Mater. 20, 415 (2008).

${ }^{21}$ H. Park, J. A. Rowehl, K. K. Kim, V. Bulovic, and J. Kong, Nanotechnology 21, 505204 (2010). 